\section{$\underset{\substack{\text { hommes } \\ \text { \& migrations }}}{ }$}

\section{Hommes \& migrations}

Revue française de référence sur les dynamiques

migratoires

1307 | 2014

L'Afrique qualifiée dans la mondialisation

\title{
Le rôle de la diaspora dans la naissance du cinéma kurde
}

Yilmaz Ozdil

\section{(2) OpenEdition \\ Journals}

Édition électronique

URL : http://journals.openedition.org/hommesmigrations/2906

DOI : 10.4000/hommesmigrations.2906

ISSN : 2262-3353

Éditeur

Musée national de l'histoire de l'immigration

Édition imprimée

Date de publication : 1 juillet 2014

Pagination : 155-160

ISBN : 978-2-919040-28-5

ISSN : $1142-852 X$

Référence électronique

Yilmaz Ozdil, « Le rôle de la diaspora dans la naissance du cinéma kurde », Hommes \& migrations [En ligne], 1307 | 2014, mis en ligne le 15 janvier 2015, consulté le 22 septembre 2020. URL : http:// journals.openedition.org/hommesmigrations/2906 ; DOI : https://doi.org/10.4000/ hommesmigrations.2906

Ce document a été généré automatiquement le 22 septembre 2020.

Tous droits réservés 


\title{
Le rôle de la diaspora dans la naissance du cinéma kurde
}

\author{
Yilmaz Ozdil
}

1 Les conflits identitaires autour de la question kurde, à leur apogée dans les années 1990-2000, ont eu pour conséquence de faire du nationalisme le principal sujet des réalisateurs kurdes. L'attribution de ce rôle propagandiste à l'art cinématographique signifie sa mise au service de la lutte politique, dont le but principal est de créer une identité nationale kurde pour un peuple divisé depuis plus d'un siècle entre quatre États (la Turquie, l'Iran, l'Irak et la Syrie).

2 Cet imaginaire d'une identité nationale kurde en construction s'impose notamment dans les films de réalisateurs kurdes exilés ou vivant au Kurdistan irakien où les films ne sont pas censurés. Dans une grande partie de ces films, toute référence à l'histoire kurde se transforme en récit de "la continuité historique" ou en "indice" de l'existence des Kurdes en tant que "nation". Cette transformation de l'histoire politique en histoire filmique se réalise particulièrement à l'aide d'un parti pris historique.

3 En raison de la complexité de la question kurde, on ne peut pas limiter l'étude du cinéma kurde à une approche purement esthétique. Depuis la réalisation du premier film en langue kurde, sur lequel nous reviendrons plus tard, des conditions politiques engendrées par le conflit kurde ont profondément marqué les configurations artistiques et la variation des discours de ce cinéma privé d'“identité officielle". Cette absence d'identité officielle, c'est-à-dire l'absence d'un État kurde, a joué un rôle plus important que l'ancienne culture des arts visuels chez les Kurdes (tels que la miniature, la gravure, la sculpture, la peinture ou la photographie) dans la détermination, la “ reconnaissance" ou encore le "rejet" d'une œuvre d'art créée par, pour, ou au nom des Kurdes. Pour la même raison, la diaspora kurde est devenue non seulement le lieu de naissance mais aussi celui du développement d'un certain cinéma kurde composé généralement de films tournés par des réalisateurs vivant hors des frontières du Kurdistan. 


\section{La naissance d'une visibilité kurde en diaspora}

4 Les conditions sociopolitiques, comme la liberté d'expression et les opportunités matérielles dans les pays d'accueil, notamment aux États-Unis et en Europe, ont permis aux élites kurdes diasporiques d'adopter l'art du cinéma au début des années 1990, soit presque un siècle après l'invention du cinématographe et son utilisation dans l'Empire ottoman et en Iran. Dans les années 1990, avec l'arrivée de centaines de milliers de réfugiés politiques kurdes en Europe, les manifestations organisées par des mouvements nationalistes kurdes contre les États dominateurs du Kurdistan faisaient régulièrement partie du paysage urbain de Paris, Berlin, Rome, Bruxelles ou encore Strasbourg ${ }^{3}$. Cette présence au sein des capitales européennes a été diffusée et reproduite par les nouveaux médias audiovisuels créés par l'élite diasporique. En 1991, soit sept ans après la mort en exil (1984) du premier réalisateur kurde Yilmaz Güney, Nizamettin Ariç, musicien exilé en Allemagne dans les années 1980 pour avoir chanté en kurde ${ }^{4}$, réalisa en Arménie son premier film, Un chant pour Beko $0^{5}$.Ce film est d'ailleurs considéré comme le premier tourné en langue kurde. Quatre ans plus tard, la première télévision kurde, Med TV, considérée comme proche du Parti des travailleurs du Kurdistan (PKK), fut créée en Europe ${ }^{6}$.

5 À l'exception notable de Yilmaz Güney, dont tous les films sont réalisés en turc, l'adoption tardive du cinéma par les Kurdes est le résultat direct de leurs conditions sociopolitiques et économiques précédant les années 1990. Alors qu'ils avaient adopté le théâtre dès $1920^{7}$, les Kurdes, mobilisés par des révoltes nationalistes depuis le début $\mathrm{du} \mathrm{XX}^{\mathrm{e}}$ siècle, n'ont pas pu s'approprier le cinéma en raison des moyens techniques et financiers plus importants exigés par la production et la distribution d'un film. Les politiques négationnistes des État-nations turc, iranien, irakien et syrien ont été l'obstacle principal pour la réalisation de films en langue kurde.

6 L'utilisation massive des médias par les mouvements nationalistes kurdes est considérée dès le début comme un "champ de bataille" par les États dominant les Kurdes. Cela s'illustre par exemple à travers l'interdiction en Turquie des médias pro-kurdes, en particulier dans les années $1990^{8}$. À cette époque, toute une panoplie de lois et de directives ont été mises en place par le gouvernement turc contre les médias prokurdes, notamment pour en empêcher la diffusion. Ces moyens juridiques s'accompagnaient d'une terrible violence visant la destruction d'établissements d'édition pro-kurdes et aussi d'assassinats politiques.

\section{Le rôle de l'élite diasporique dans la naissance du cinéma kurde}

7 Dans le milieu du cinéma kurde, notamment dans la diaspora, on assiste à l'émergence d'une nouvelle élite artistique en relation permanente avec les élites politiques kurdes. Même si leurs relations ne prennent pas systématiquement la forme d'une adhésion explicite aux organisations politiques, les artistes appartiennent parfois aux cercles restreints de ces élites. Ainsi, il est devenu presque banal de voir défiler dans les génériques des films kurdes tournés au Kurdistan irakien les noms de personnalités ou de membres de l'élite administrative et gouvernementale. Le générique du film Dol ou la vallée des tambours (2007) de Hiner Saleem en est un exemple emblématique. 
8 La dispersion géographique des réalisateurs kurdes montre, d'une part, ce lien étroit entre le cinéma et la question politique kurde et, d'autre part, la raison essentielle de la forte volonté de "victimisation" et de "kurdicité" dans les films tournés. Tous touchés par les conséquences tragiques de la récente histoire kurde, une grande partie des réalisateurs vivent actuellement dans des pays occidentaux comme l'Allemagne, la Belgique, la France, la Suisse, la Suède, la Norvège, l'Angleterre ou les États-Unis.

Bien avant la réalisation d'Un chant pour Beko de Nizamettin Ariç, le réalisateur kurde Yilmaz Güney avait quitté la Turquie en 1981 après avoir passé une dizaine d'années dans les prisons turques pour son engagement politique. Durant les trente-deux ans de sa carrière cinématographique, Güney dut réaliser tous ses films en langue turque en raison de l'interdiction officielle de l'usage public de la langue kurde en Turquie. À propos de son célèbre film Le Troupeau (1978), il s'exprime ainsi : "Le Troupeau, en fait, c'est l'histoire du peuple kurde, mais je n'ai même pas pu utiliser la langue kurde dans ce film. Si on avait utilisé le kurde, tous ceux qui ont collaboré à ce film auraient été mis en prison ${ }^{10}$."

Bien qu'il ait été considéré comme un "réalisateur turc" jusqu'à sa mort ${ }^{11}$, Güney avait en réalité été déchu de la nationalité turque en $1982^{12}$, soit deux ans avant sa mort, pour “ trahison de la patrie". Sa nationalité turque lui fut discrètement "rendue" seulement en 1993. Ce n'est cependant qu'en 2009 que les médias turcs révélèrent cette réalité13. Concernant Ahmet Zirek, qui a notamment interprété le personnage de Jaffer dans le dernier film de Yilmaz Güney, Le Mur (1983), il est toujours apatride ${ }^{14}$ et vit actuellement à Paris. Il raconte son histoire d'exil dans son film Pari(s) d'exil, tourné en 2009 et sorti le 8 avril 2013.

\section{L'identité kurde, entre opportunité et obstacle}

11 L'identité kurde d'un réalisateur, parfois non revendiquée par lui-même, n'est pas suffisante pour qualifier un film de "film kurde". Beaucoup de réalisateurs kurdes vivant dans différentes parties du Kurdistan sont considérés comme non kurdes. Parmi ces réalisateurs, nombreux sont ceux qui travaillent dans l'industrie cinématographique turque, dont les films n'ont aucune affiliation identitaire avec leurs origines. Bahman Ghobadi, Kurde iranien possédant toujours la nationalité iranienne, a dû quitter son pays natal après avoir réalisé Les Chats persans (2009), en raison d'une interdiction de tourner.

D'après Hamid Dabashi, historien du cinéma iranien, le fait que les réalisateurs kurdes aient une double identité n'empêche pas de considérer leurs films comme appartenant au cinéma kurde. Dabashi souligne: "De par leur rapport à la langue, à la culture, à la politique, à leurs aspirations nationales et aux réalités quotidiennes, la topographie visuelle de ces réalisateurs incarne la réalité kurde et nous transmet beaucoup de choses sur cette réalitée ${ }^{15}$." Particulièrement dans la diaspora, cette double appartenance n'est pas toujours un obstacle, elle peut même devenir un atout pour financer leurs projets. Bien que ni dans les pays d'accueil, ni au Kurdistan, leurs films ne dépassent généralement pas les 100000 entrées. La plupart de ces réalisateurs arrivent à les produire grâce à d'importantes contributions publiques, notamment européennes, qui s'ajoutent à celles $\mathrm{du}$ gouvernement régional $\mathrm{du}$ Kurdistan irakien. Ces films bénéficient d'un financement accordé par des organisations comme le Centre national cinématographique français ( $\mathrm{CNC}$ ) ou Eurimages, des chaînes de télévision comme Arte et Canal +, et ils profitent également de la contribution de plusieurs maisons de 
production et de sociétés de distribution européennes. À titre d'exemple, le film My

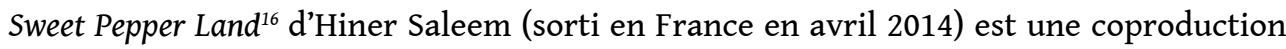
française, allemande et kurde. Plusieurs festivals internationaux ont participé à la production du film La Vie de Jano Rosebiani (2002) ${ }^{17}$.

\section{La reconnaissance des festivals internationaux} festivals internationaux dans la structuration des discours sociopolitiques sur la " kurdicité" dans les films kurdes. Ce phénomène peut s'expliquer par l'absence d'un marché du cinéma au Kurdistan, elle-même liée à l'absence d'un État kurde reconnu. Ne pouvant pas commercialiser leurs films au Kurdistan, la plupart des réalisateurs kurdes réalisent aujourd'hui leurs films d'abord pour les festivals internationaux, les marchés étrangers et les spectateurs non kurdes.

construction des histoires filmiques aux discours médiatiques des réalisateurs, cette absence de marché du cinéma au Kurdistan influence toutes les dimensions du processus de production des films kurdes. Cette transformation des pays étrangers en destination principale du cinéma kurde doit cependant aussi être considérée comme le résultat d'un choix politique visant à mobiliser l'opinion publique internationale sur la question kurde. La distinction entre les Kurdes et leurs adversaires politiques, à travers le traitement de sujets "plus humains et non violents", se présente aujourd'hui comme une nécessité dans le cinéma kurde. Dans le choix du sujet et de l'esthétique d'un projet, sans aller jusqu'à parler des origines kurdes de Saladin, un certain cinéma kurde peut s'affirmer et se détacher du cinéma officiel des États où vivent les Kurdes. Depuis Un chant pour Beko, dont la date de réalisation coïncide avec l'intensification des apparitions de Kurdes irakiens dans les médias internationaux, la grande majorité des réalisateurs kurdes construisent leurs histoires filmiques sur cette base politique.

\section{Filmer les Kurdes depuis la diaspora}

Bien que la question kurde ainsi que le contexte sociopolitique et économique des Kurdes soient évoqués dans la plupart des films kurdes, il existe de véritables différences entre les films tournés au Kurdistan et ceux tournés en diaspora. Dans les premiers, les références à la question kurde sont bien visibles. La structure narrative de ces films évolue en général d'un rapport politique conflictuel entre des personnages kurdes, souvent présentés comme des "victimes", et leurs antagonistes (non kurdes), souvent présentés comme des "oppresseurs". Dans leur univers diégétique, la "kurdicité" se transforme en intériorité/esprit identitaire dominant, par rapport à "l'Autre" conçu souvent comme "extériorité menaçante". Autrement dit, par des rapports conflictuels, la "kurdicité" devient un système de valeurs morales, selon lequel le "mal" est identifié à l'“Autre" (Turcs, Arabes, Persans ou collaborateurs kurdes) et le "bien" au "nous" (Kurdes).

16 À la différence de ces films tournés au Kurdistan traitant la question kurde sous un angle politique, dans les films tournés dans la diaspora, la "kurdicité" s'exprime soit comme une identité périphérique confrontée à d'autres identités, soit comme un univers clos dans lequel le personnage kurde est isolé. Dans la majorité de ces films, le 
rapport à l'identité kurde se fait à travers le traitement des problèmes socioéconomiques liés à l'immigration. Par exemple, les sujets de l'exil et de la vie en diaspora apparaissent comme les thèmes principaux de beaucoup de films ${ }^{18}$. Parmi ces films, on peut citer Pari(s) d'exil (2009) d'Ahmet Zirek, La petite liberté (2003) de Yüksel Yavuz, Vinterland (2007) et Befor Snowfall (2013) de Hisham Zaman, Vive la mariée... et la libération du Kurdistan (1997) ${ }^{19}$ et Si tu meurs je te tue (2010) de Hiner Saleem, Suivre le duvet (2004) de Nuray şahin, En garde (2004) d'Ayşe Polat, ainsi que David et Layla (2005) de Joy Jonroy.

Les films kurdes réalisés en diaspora, notamment ceux de Hiner Saleem, traitent la “ kurdicité" par rapport à une "modernité occidentale" dont l'identité kurde ne fait pas partie. Nous pouvons observer les traces de cette mise en conflit identitaire dans notamment Vive la mariée... et la libération du Kurdistan et Si tu meurs je te tue que Hiner Saleem a réalisé à Paris. D'une façon générale, la "kurdicité" est orientalisée dans ces deux films à travers un rapport d'opposition morale et culturelle entre les "origines orientales" des personnages kurdes et l'identité occidentale (française) en devenir. Le traitement de la question des "droits de la femme", auxquels Hiner Saleem fait souvent référence ${ }^{20}$, s'expose comme une stratégie de mise en avant de certaines "valeurs occidentales" face à la "culture orientale" kurde. Cette même question fait partie, par exemple, d'une certaine image péjorative des Kurdes reproduite pendant presque un demi-siècle (depuis des années 1950 jusqu'à la seconde moitié des années 1990) par le cinéma turc.

Cette variation thématique et discursive des films kurdes tournés en diaspora est liée à la réalité sociale des communautés diasporiques kurdes ainsi qu'aux codes socioéconomiques des marchés cinématographiques dont dépend le cinéma kurde. Cela montre que, même si le regard porté sur la question kurde apparaît comme homogène dans leurs films, le langage cinématographique des réalisateurs kurdes est marqué par les différentes sociétés au sein desquelles ils produisent leurs films, par les conditions sociopolitiques et économiques, par les systèmes de production qui leur sont propres, ainsi que par des publics très sélectifs. Cela signifie que, quels que soient leurs discours médiatiques sur la cause kurde, les discours cinématographiques des réalisateurs sur leur propre société peuvent varier selon les conditions réelles dans lesquelles ils produisent et commercialisent leurs films.

\section{NOTES}

1. Voir à ce sujet François Hartog, Évidence de l'histoire, Paris, Gallimard, 2005, et Jacques Le Goff, Histoire et mémoire, Paris, Gallimard, 1988.

2. Benedict Anderson, L'Imaginaire national. Réflexions sur l'origine et l'essor du nationalisme, Paris, La Découverte/Syros, 2002.

3. Oliver Grojean, "L'engagement politique à distance", in Colloque de la Section d'études internationales (SEI) de l'Association française de science politique (AFSP), "Les solidarités transnationales", 2003.

4. Voir à ce sujet, Burçin Demirtola Çelik, Sürgünde Kürtler, Istanbul, Aram Yayınları, 2013. 
5. Un chant pour Beko (1992) de Nizamettin Ariç est considéré par plusieurs chercheurs comme le premier film kurde tourné en langue kurde. Voir Yilmaz Ozdil, "La construction visuelle des identités kurdes au cinéma", thèse de doctorat, université Paris-III-Sorbonne-Nouvelle, 2013.

6. Cette première chaîne de télévision (Med TV) est créée par le PKK en diaspora en 1995, dans une période sanglante de lutte armée contre le gouvernement turc. Si l'on ne considère pas la chaîne de télévision de Kirkuk, fondée en 1967 par le régime bassiste irakien et ayant commencé à diffuser en kurde au début des années 1970, comme une télévision kurde, Med TV fut la première chaîne kurde créée par une organisation politique kurde. Aujourd'hui, dans toutes les parties du Kurdistan et en diaspora kurde, il y a plus de trente chaînes de télévision kurdes toutes reliées, d'une façon ou d'une autre, à une organisation politique kurde. Voir Amir Hassanpour, "Satellite footprints as national borders: Med TV and the extraterritoriality of state sovereignty", in Journal of Muslim Minority Affairs, vol. 18, n 1, 1998, pp. 53-72.

7. Voir Amir Hassanpour, Kürdistan'da Milliyetçilik ve Dil: 1918-1985, Istanbul, Avesta Yayınları, 1997, pp.495-498. Il faut ajouter que le théâtre est adopté par les mouvements nationalistes kurdes, pas seulement dans un but de propagande, mais également comme un loisir alternatif. Par exemple, après le Parti démocrate du Kurdistan irakien (PDK) et l'Union patriotique du Kurdistan en Irak (UPK), on voit aujourd'hui, au sein même du PKK, des combattants interprétant des textes politiques dans une "pièce comique", en plein milieu de la montagne. De la même façon, dès le lancement de la première télévision kurde, Med TV, on assiste à l'apparition du groupe de théâtre Koma Ehmedê Xanî (Group d'Ehmedé Xanî). Les pièces interprétées par ce groupe furent diffusées et rediffusées pendant longtemps par Med TV et Roj TV.

8. Voir Isabelle Rigoni, "Med TV dans le conflit kurde", in Confluences Méditerranée, n 34, Paris, L'Harmattan, 2000, pp. 45-51.

9. Voir Pascal Pignol, "Le travail psychique de victime : essai de psycho-victimologie", thèse de doctorat, université de Rennes-II-Haute-Bretagne, 2011.

10. Extrait de l'interview de Chris Kutschera avec Yilmaz Güney, publiée en janvier 1983 dans Middle East Magazine, disponible sur : http://www.chris-kutschera.com/Yilmaz\%20Guney.htm

11. Par exemple, dans le film Chambre 666 (1984) de Wim Wenders, le réalisateur allemand dit qu'il vient de rencontrer un réalisateur turc qui a fui son pays et qui avait peur de venir tourner la séquence parce qu'il était suivi par des services de renseignement.

12. Voir Tolga Akıner, "Yılmaz Güney vatandaş olmuş, kimseler duymamış !”, in Radikal, 9 janvier 2009 [En ligne ] :

http://www.radikal.com.tr/turkiye/yilmaz_guney_vatandas_olmus_kimseler_duymamis-916222.

13. Ibid.

14. Pour plus d'informations, voir le site officiel du film : http://www.parisdexil.com/

15. Hamid Dabahsi, “Önsüz” (préface), in Kürt Sineması : Yurtsuzluk, Stnır ve Ölüm, op. cit., pp. ix-x.

16. Voir http://www.allocine.fr/film/fichefilm-224106/casting/\#production.

17. Dans le générique du film Jiyan de JanoRosebiani, il est indiqué que le gouvernement régional du Kurdistan (KRG), le Festival international du film de Rotterdam (Fonds Hubert Bals), le Festival international de Locarno et le Festival du film de Göteborg ont participé à la production de ce film, dont le sujet porte sur le massacre d'Halabja par le régime baasiste de Saddam Hussein.

18. Voir Yilmaz Ozdil, "La construction visuelle des identités kurdes au cinéma", op. cit.

19. Voir à ce sujet Salih Akin, "Language and cultural contact: Vive la mariée et... la libération du Kurdistan", in Verena Berger, Miya Komori (dir.), Polyglot Cinema: Migration and Transcultural Narration in France, Italy, Portugal and Spain, Wien, LIT, 2010, pp. 111-124.

20. Dans une émission télévisée présentée sur Canal +, Hiner Saleem dit avoir fait ce film ( $S i$ tu meurs je te tue) "pour rendre un hommage à la femme musulmane". Un extrait de cette interview est disponible sur: http://www.canalplus.fr/c-cinema/c-emissions-cinema-sur-canal/pid6301interviews-cinema.html?vid=444868. 


\section{AUTEUR}

YILMAZ OZDIL

Docteur en cinéma de l'université Paris-III-Sorbonne-Nouvelle 